\title{
Le marché des armes après la guerre froide et les pays les moins développés
}

Frederic S. Pearson

\section{(2) OpenEdition}

\section{Journals}

Édition électronique

URL : http://journals.openedition.org/conflits/123

DOI : $10.4000 /$ conflits. 123

ISSN : $1777-5345$

Éditeur :

CCLS - Centre d'études sur les conflits lilberté et sécurité, L'Harmattan

Édition imprimée

Date de publication : 17 janvier 1991

ISSN : 1157-996X

\section{Référence électronique}

Frederic S. Pearson, «Le marché des armes après la guerre froide et les pays les moins développés »,

Cultures \& Conflits [En ligne], 04 | hiver 1991, mis en ligne le 31 décembre 2002, consulté le 30 mars 2021. URL : http://journals.openedition.org/conflits/123 ; DOI : https://doi.org/10.4000/conflits.123

Ce document a été généré automatiquement le 30 mars 2021.

Creative Commons License 


\title{
Le marché des armes après la guerre froide et les pays les moins développés
}

\author{
Frederic S. Pearson
}

L'impact des changements contemporains

Vers une restriction des transferts d'armement?

La fin de la guerre froide en 1989, la guerre du Golfe ensuite et la notion même de "nouvel ordre mondial" ont eu pour effet combiné de compliquer passablement le fonctionnement du marché international de l'armement et d'y jeter une certaine confusion. Une demi décennie de baisse relative des ventes d'armes au tiers-monde, de réduction des dépenses de défense et d'augmentation du nombre des exportateurs éventuels ont conduit à l'émergence d'un véritable "marché acheteur". Il est ainsi apparu que plus de coopération entre les grandes puissances et toute initiative en faveur du règlement des conflits régionaux aussi déstabilisateurs qu'interminables (Angola, Afghanistan, Amérique centrale, Kampuchéa et Irak-Iran) entraîneraient une baisse des commandes d'armement. Les problèmes résultant de l'important endettement et du marasme économique persistant du tiers-monde n'ont pu que renforcer ces perspectives. De plus le ralentissement des combats en maintes régions et la saturation des besoins en matériel de défense ont entraîné une chute sévère de la production et des ventes d'armement chez des acteurs de "second rang" (Brésil, Israël,), menaçant ainsi, à terme, leur statut d'exportateur de matériel militaire ${ }^{1}$.

Les perspectives d'une restriction des transferts d'armement qui ne semblaient plus d'actualité depuis l'échec des pourparlers du CATT initié par le président Carter ont été réouvertes à l'occasion de la réponse apportée par la communauté internationale à l'invasion irakienne du Koweï. Tout le monde est tombé d'accord en effet pour une réduction du flot des armes destinées à des zones aussi instables que le Moyen-Orient. Lé président Bush, le Premier ministre Mulroney, les leaders européens ne se voyaientils pas accusés d'avoir contribué au développement d'une machine de guerre surdimensionnée et de s'être mis en situation de combattre leur propre système 
d'armes dans des conflits lointains? Quant aux responsables soviétiques, ils paraissaient fermement décidés à réorienter un système basé sur les besoins de la défense vers une économie plus productive. A dire vrai, le Moyen-Orient avait déjà cédé à l'Asie du Sud sa place de premier importateur du tiers-monde. En outre dès la fin de la guerre du Golfe, plusieurs états pétroliers de la région ont eu à faire face à d'importants besoins de reconstruction civile et d'environnement au détriment des crédits consacrés naguère à l'achat d'armement ${ }^{2}$. I1 n'est jusqu'à la Chine qui semble avoir modéré ses principaux besoins d'armement à l'issue du conflit Irak-Iran. Ainsi peut-on avancer à titre d'hypothèse que la guerre du Golfe et que le nouveau rôle joué par les Nations Unies en faveur de la paix ont fait naître l'idée nouvelle d'un régime international d'inspection et de contrôle des transferts d'armement à destination des régions où le foisonnement des conflits est à craindre.

Cependant, bien que ces perspectives de limitation toute relative des armements soient encore à l'ordre du jour, des incertitudes et des opinions contraires se sont manifestées. Des parlementaires plaidant pour le contrôle des armements, des analystes et des responsables politiques comme Bush et Mulroney ont certes continué à recommander des formules allant de l'adoption d'un moratoire sur l'exportation de matériel militaire au Moyen-Orient à la conclusion d'un traité américano-soviétique ou à un traité multilatéral visant à interdire ou à réduire un tel commerce, mais d'un autre côté l'administration Bush a poussé à la vente massive d'armement supplémentaire à ses partenaires de la coalition anti-irakienne à titre de récompense et pour leur protection. Pour la première fois depuis 1974, des propositions sujettes à controverse ont été avancées en vue d'autoriser l'Export-Import Bank à accorder des prêts garantis pour l'achat de matériel militaire américain, non plus seulement aux principaux alliés des Etats-Unis ou à des clients tels qu'Israël comme c'était initialement prévu, mais aussi, éventuellement, à d'autres bénéficiaires que l'intérêt national recommande au Président ${ }^{3}$.

Mis à part les ventes d'armes au Moyen-Orient, estimées à environ 20 milliards de dollars, Washington a, dit-on, négocié pour plus de 13 milliards de dollars de contrats de ventes d'armes avec des clients latino-américains, africains et asiatiques en 1991. Alors que la réduction des exportations peut aboutir dans certains cas à une plus grande sécurité, le Département de la défense en particulier, a pour ainsi dire calculé que la fourniture d'armes à des clients favorisés, en vue de la stabilité d'une région, était une démarche plus efficace d'un point de vue militaire, plus acceptable politiquement et plus lucrative économiquement (pour les entreprises d'armements et les budgets de défense américains) ${ }^{4}$. En fait, si I administration a cherché à faire passer des accords de contrôle d'exportations d'armement et si une pression politique s'est exercée en vue de faire imposer par le Congrès des restrictions à ces exportations, il était surtout question de certaines technologies sensibles, de commerce d'armes EstOuest, de prolifération balistique, nucléaire, chimique et bactériologique ("CBW") et de l'exportation de super-ordinateurs 5 . On entendait alors dire communément que si ce n'était pas les Américains qui vendaient des armes, la clientèle s'adresserait à des fournisseurs concurrents et que, de toute façon, un pays qui le voulait trouverait toujours le moyen de s'armer ${ }^{6}$.

De manière similaire, bien que les dépenses de défense soviétique aient accusé une baisse estimée à environ $6 \%$ pour chacune des deux dernières années, un pressant besoin de devises fortes a entraîné pour les états de l'ancien bloc de l'Est quelques arrière-pensées quant à la restriction de leurs exportations militaires. La 
Tchécoslovaquie, par exemple, a décidé en 1991 de fournir des tanks à la Syrie malgré l'intention du président Havel d'interdire tout transfert d'armement.

L'Union soviétique a continué de vendre des armes tels que le MIG-29, à l'Iran et à la Syrie. Bien que la question sur l'opportunité de l'exportation d'armes ait donné lieu à de furieuses empoignades à Moscou, et malgré le plongeon enregistré dans la commercialisation des armes soviétiques durant la guerre du Golfe, les autorités veulent, visiblement, continuer à exporter aussi longtemps que Washington le fera ${ }^{7}$. On dit aussi de la Chine qu'elle a poursuivi plus avant ses efforts dans la vente de missiles balistiques de moyenne portée ${ }^{8}$.

La contribution des états de l'Europe occidentale à une hypothétique réduction du commerce des armes a dépendu, dans une certaine mesure, d'une improbable intention des Etats-Unis et de l'OTAN de remédier à la stagnation du marché par un accroissement de leurs propres importations. On a cru même pouvoir s'y attendre lorsqu'on a vu l'ambassadeur américain auprès de l'OTAN suggérer, en guise d'alternative aux ventes d'armes au tiers-monde une augmentation du commerce des armes entre pays membres de l'alliance grâce à l'abaissement de leurs tarifs douaniers. Cependant les relations commerciales Nord-atlantique sont loin d'être clarifiées à l'orée de 1992 alors que la tentation de commercer avec le tiers-monde et d'y proposer des armes de haute technologie est devenue plus forte après les prétendus succès de la guerre du Golfe, notamment les attaques réussies de missiles contre Israël. Alors que l'Allemagne semble déterminée à passer outre les pénalités attachées aux exportations illégales, la volonté de la Communauté européenne en tant que telle pour freiner la promotion des ventes d'armes en dehors de l'OTAN apparaît elle-même problématique. Ainsi la guerre du Golfe a-t-elle révélé de profondes divergences entre les états de la Communauté sur la politique de défense à suivre, la Belgique refusant notamment de s'approvisionner auprès de la Grande-Bretagne. A l'inverse la guerre a dans le même temps accentué la pression de l'opinion publique et des élites en faveur d'une coopération renforcée en matière de coordination de la sécurité européenne9. II en ait résulté essentiellement une série de négociations bilatérales entre les principaux états européens, notamment la France, la Grande-Bretagne et l'Allemagne en vue de déterminer quelle structure conviendrait le mieux aux prises de décisions en matière de politique de défense, et entre autre pour concilier les intérêts de l'OTAN avec ceux de la CEE, de la CSCE et de l'UEO ${ }^{10}$.

D'une certaine manière, compte tenu des intérêts contradictoires entre le maintien simultané de la stabilité régionale et les ressources résultant du commerce des armes, l'éventualité d'une restriction des transferts d'armement reste faible: au-delà du consensus portant sur la limitation de la diffusion des technologies avancées les plus dangereuses, elle dépend aussi du désir et de la capacité des états importateurs de trouver des garanties alternatives de sécurité, d'acheter des armes et de convaincre leurs fournisseurs de leur procurer ces dernières à crédit. Certains importateurs d'armes, tels le Pakistan, ont apparemment tiré comme leçon de la guerre du Golfe que seules les armes les plus modernes, les plus "intelligentes", voire les plus "furtives" peuvent assurer la sécurité des pays modernes. Les dirigeants israéliens de leur côté ont affirmé depuis longtemps que la qualité des forces peut combler les désavantages quantitatifs. Selon le Premier ministre pakistanais "l'aspect militaire (de la guerre) du Golfe a clairement montré que s'en prendre au potentiel scientifique, technologique et de recherche d'un pays ainsi qu'à ses réalisations les plus modernes en matière de 
défense entraîne pour ce pays un véritable désastre Le défi technologique est un phénomène permanent"11.

De telles opinions, bien entendu surestime l'utilité des systèmes d'armes très avancés dans l'environnement électronique du champ de bataille dès lors que fait défaut la maîtrise des systèmes de surveillance de l'espace, de commande et de contrôle. En termes clairs cela signifie que maints gouvernements de pays sous-développés, spécialement ceux qui sont engagés dans des conflits régionaux chroniques, peuvent atteindre le plus haut sommet de la technologie militaire, leur véritable capacité n'en reste pas moins limitée à cause de leurs insuffisances en matière de ressources budgétaires, scientifiques et humaines. En outre, comme Andrew Pierre l'a remarqué, une relance massive de la course aux armements ne devrait pas concerner le MoyenOrient puisqu'en 1991 seuls l'Irak et le Koweït y ont perdu des quantités significatives d'armement ${ }^{12}$. Néanmoins la mise en service de versions améliorées de missiles balistiques et de croisière, de projectiles guidés avec précision, ainsi que de petits sousmarins indétectables et de mines, promettent dans un avenir relativement proche une augmentation des capacités meurtrières de nombreux pays sous-développés ${ }^{13}$.

Les changements intervenus dans le système international de l'après-guerre froide rendent illusoires l'existence d'un réel pouvoir des acheteurs sur le prix des armes, mais, comme pour les pays exportateurs, les tendances sont contradictoires. Le déclin de la compétition entre les grandes puissances limite pour les acheteurs la possibilité de faire jouer la concurrence entre leurs fournisseurs. Les clients traditionnels des superpuissances, tels que le Pakistan et la Syrie, peuvent moins compter sur le patronage systématique des Etats-Unis ou de l'Union soviétique quoique d'une certaine façon les approvisionnements aient toujours été sporadiques, spécialement en temps de crise, selon que les superpuissances cherchaient soit à soutenir, soit à contenir leurs clients. Mais dans le même temps, les grandes puissances sont aujourd'hui en compétition aussi bien pour obtenir une part du marché des armes que pour bénéficier d'avantages politiqués. Depuis que la richesse s'est faite rare, les acheteurs capables de payer comptant ou prêts à passer d'importantes commandes d'armes suscitent entre les fournisseurs une concurrence considérable qui se traduit notamment par des offres de compensation sous forme de transferts technologiques et de possibilités de crédits. De plus comme les accords entre les plus grandes puissances ont stabilisé le paysage militaire européen (CFE, par exemple) les surplus d'armes ont augmenté, rendant plus aisées les exportations de pays tels que l'URSS ou la Tchécoslovaquie ${ }^{14}$ et peut être aussi en définitive des pays d'Europe de l'Ouest. Dès lors la question cruciale, pour l'avenir de la stabilité régionale, est de savoir si les ventes d'armes seront réduites, simplement limitées aux seuls clients les plus industrialisés du tiers-monde ou, au contraire, largement répandues parmi les pays les moins développés. On peut s'attendre, à titre d'hypothèse de travail, à une plus grande limitation du commerce des armes dans les zones où les acheteurs semblent économiquement à bout de force et où les probabilités de guerre sont relativement fortes. L'apparition d'un marché de l'armement d'occasion Afin de tester une telle hypothèse, il est important de connaître plus précisément l'évolution des préférences et des politiques d'équipement des pays les moins développés, en particulier de ceux qui sont soumis aux contraintes économiques, militaires et technologiques les plus lourdes. On accordera aussi beaucoup d'attention aux grands importateurs du tiers-monde: les plus puissants, les plus riches en ressources, les plus "avancés", comme l'Irak, l'Iran et l'Inde, pays qui totalisent à eux seuls une grande partie des achats des grands systèmes majeurs. Les pays sous- 
développés, les plus faibles, les plus pauvres, les moins bien dotés, constituent en effet un marché fluctuant dont on comprend moins bien les mécanismes et dont l'importance pourrait s'accroître dans les années à venir. Nombre de ces états (notamment ceux de la Corne de l'Afrique, de l'Amérique centrale et de l'Asie du Sud) ont longtemps été en guerre et souhaitent maintenant jouer des rôles militaires régionaux de premier plan.

La diminution des conflits entre grandes puissances aurait pu entraîner un accroissement des difficultés d'approvisionnement pour les pays sous-développés. Toutefois la perception des menaces s'est élevée, notamment pour les états membres de l'ASEAN pendant la guerre du Golfe. Dès lors deux questions se posent : premièrement, les états doivent-ils avoir recours à des moyens militaires pour jouer un rôle plus actif au niveau régional? Deuxièmement parviendront-ils à combler leur retard technologique? L'une des conséquences les plus frappantes de l'évolution récente de la hiérarchie technologique internationale réside dans l'apparition de certains pays sousdéveloppés exportateurs sur le, ou plutôt, sur les marchés des armes. Il existe de ce fait un véritable marché de l'armement d'occasion; d'ailleurs certaines armes, importées des grands pays industrialisés, sont réexportées après avoir été au préalable modifiées, modernisées ou tout simplement copiées. Une limitation significative des exportations des grandes puissances exportatrices n'empêchera donc pas la Chine, le Brésil, l'Argentine, Israël, les deux Corée, Taïwan, et l'Afrique du Sud d être vraisemblablement capables de poursuivre leurs exportations de matériels d'occasion ${ }^{15}$ La symbolique du nucléaire dans le tiers monde

Comprendre les objectifs poursuivis en matière d'armement par les pays sousdéveloppés nous oblige à revenir sur leur place dans la hiérarchie du système international et des sous-systèmes régionaux. Pour des raisons de puissance, d'histoire et de géopolitique (les menaces militaires étant constituées par les mouvements insurrectionnels et d'oppositions internes ${ }^{16}$, ces pays se sont consacrés au maintien des équilibres régionaux plutôt qu'internationaux. Leur tendance à associer à leur dispositif de sécurité des puissances extra-régionales (par des traités d'amitié et d'assistance) a été bouleversé par la transformation des relations entre les superpuissances Même si l'apparition de tels liens était inévitable, les pays sousdéveloppés ont toujours tenu à afficher au moins symboliquement, leur indépendance. La possession d armes était dans cette perspective un élément crucial, dans la mesure où les technologies militaires provenaient de firmes prestigieuses, mais également parce que le développement des capacités locales de production lié à certains contrats (comme les accords de production sous licence) renforçaient leur indépendance.

Ayoob affirme par exemple que les armes nucléaires constituent pour les états du tiersmonde souffrant d'une douloureuse impuissance une sorte de moyen symbolique pour s'affirmer auprès des grandes puissances et peser sur leur politique. Comme l'a montré jadis la victoire du Japon sur la Russie, les pays asiatiques peuvent être autre chose que des perdants. Le renforcement de la position de la Chine (ou de l'Inde) grâce à son statut de puissance nucléaire, sa position actuelle d'exportateur d'armes de tout premier plan, soulignent l'importance du rôle joué par la technologie, non seulement dans l'allégement du déficit de ses échanges avec l'étranger, mais également dans la dissipation du sentiment de frustration et de découragement. Si le niveau de l'armement n'est pas important comme le prétendent l'Algérie et quelques autres pays parmi les moins développés, si le statut des armes n'est pas important, pourquoi de grandes puissances telles que les Etats-Unis surveillent-ils alors de si près leurs 
programmes de développement et s opposent-ils si farouchement à celui de l'Irak ${ }^{17}$ ? En réalité la question des armes fait partie intégrante de la sécurité, au même titre que la stratégie économique, même pour des états tel que le Brésil qui n'ont aucun ennemi extérieur ou intérieur apparent ${ }^{18}$. La sécurité en arrive, dans un langage "déconstructionniste", à avoir un sens nouveau et plus largement politique en termes de statut international, d'influence et de contingences futures. Ce n'est donc pas un hasard si parmi les états importateurs d'armes, les plus demandeurs sont aussi ceux qui, s'efforçant d'avoir un projet économique, développent aussi une capacité de production d'armes propre ainsi qu'un potentiel nucléaire ${ }^{19}$.

Nous en sommes arrivés à associer utilisation symbolique de la technologie militaire et pays nouvellement industrialisés, qui sont aujourd'hui les puissances dominantes du tiers-monde. Les autres après tout ne peuvent généralement s'offrir une telle panoplie. Ils ont signé le Traité de non-prolifération, renonçant de ce fait à leur désir d'acquérir des armes nucléaires. Toutefois, nous n'avons pas complètement exploré le rôle plus général de l'armement dans les stratégies de sécurité des pays les moins développés. Alors qu'il serait vain pour de tels états de se lancer dans des programmes nucléaires coûteux, ils peuvent très bien montrer un intérêt comparable pour les armes conventionnelles, officiellement pour répondre à des raisons de sécurité prises au sens le plus large.

Nous nous proposons ici, de revoir les dernières politiques d'acquisition d'armement de certains des pays les moins développés représentatifs de cette tendance, choisis dans chaque continent, afin de déterminer s'il existe des modèles permettant de prédire leurs futures démarches en matière d'armement. Quoique les variables indépendantes suivantes n'aient pas grand chose à voir avec la quantité des armes importées par les pays les moins développés ${ }^{20}$, elles ne sont pas sans lien avec la qualité ou la nature de l'armement recherché à l'extérieur ou fabriqué localement.

Ce sont :

1) une expérience de guerre récente, 2) la perception d'une menace tant extérieure qu'intérieure, 3) la taille des pays et leur richesse, 4) leur localisation géopolitique.

Les unités de notre échantillon ont été choisi de manière à faire apparaitre différentes valeurs de ces variables. Vers un modèle d'analyse des politiques d'armements des pays les moins développés

Des politiques d'approvisionnement diversifiées

Les politiques de défense mises en oeuvre depuis 1967 en Afghanistan, Algérie, Chili, Colombie, Malaisie, Nigéria, Sénégal, Sri Lanka, Thaïlande, Yémen et Zimbabwe ont été examinées en utilisant les constatations faites par le Defense and Foreign Affairs Handbook $^{21}$ : Bien sûr, il n'existe aucune certitude pour que le type d'armes acquis auprès de multiples fournisseurs, au cours de ces cinq dernières années, constitue un élément indicatif sur les politiques à venir de ces états. Mais les plans d'approvisionnement prouvent que les liens établis avec les fournisseurs tendent à influencer les décisions futures (remplacements des matériels et pièces de rechange) et à orienter la planification de la politique de défense, prévue généralement pour des périodes de 5 ans. Pour des pays qui sont encore activement engagés dans des conflits d'importance tels l'Afghanistan, la Colombie, le Sénégal, le Sri Lanka, et dans une certaine mesure la Thaïlande, les efforts sont dirigés sur des équipements destinés à des objectifs militaires bien spécifiques, malgré les contraintes imposées pour le choix de ces armes par les difficultés économiques. 
La Colombie par exemple, qui s'était engagée dans des opérations anti-drogue et antiinsurrection alors qu'aucune menace extérieure n'était visible, mise à part les tensions épisodiques avec le Venezuela, a axé principalement ces approvisionnements sur de l'armement léger et sur les moyens de communication. La configuration géographique du terrain et les exigences logistiques expliquent les préférences accordées aux blindés légers (transports de troupes et véhicules blindés) par rapport aux chars lourds. L'acquisition de "petits" matériels y compris des avions de chasse légers demeure une priorité. Ainsi la Colombie a échangé du charbon avec Israël pour le contrat des "Kfirs" en 1988, portant sur des hélicoptères, des missiles antiaériens, des moyens antichars, du matériel de surveillance aérienne, des patrouilleurs et des vedettes fluviales.

Le Sénégal, quant à lui, confronté à de sérieuses difficultés économiques, à des conflits ethniques et à des conflits frontaliers avec la Guinée, la Mauritanie et la Guinée-Bissau, a poursuivi une politique d'armement identique. S'il a participé à la coalition des forces alliées lors de la guerre du Golfe en 1990, c'est en partie peut-être pour renforcer les liens avec ses principaux fournisseurs, les Etats-Unis et la France notamment, alors que Washington avait tenté auparavant de lier à l'interdiction de la drogue son assistance militaire au Sénégal . Malgré les conflits dans lesquels il est engagé, le Sénégal a néanmoins réduit le budget de ses forces armées en 1990-1991, reflétant ainsi les bouleversements économiques dues à l'échec de la confédération avec la Gambie. Ainsi la satisfaction des besoins en armement de ces états reste-t-elle tributaire des relations instables et incertaines qu'ils entretiennent avec leurs fournisseurs. Ils recherchent des sources d'armement fables, à prix réduits, évitant le plus possible de tomber dans une relation de dépendance exagérée vis-à-vis de fournisseurs uniques susceptibles d'intervenir dans leurs prises de décision ou de leur imposer une gestion particulière du lourd fardeau que représente la dette extérieure. Une de leurs préoccupations est d'explorer un large marché en vue de choisir les fournisseurs d'armes les moins chers. Le Sri Lanka entretient traditionnellement des liens privilégiés dans le domaine de la défense avec la Grande-Bretagne, toutefois il bénéficie également de l'assistance militaire des Etats-Unis, de l'Inde, du Pakistan et d'Israël, tout en achetant des armes à la Grande-Bretagne, à Singapour, à la Corée du Sud, au Pakistan, à la Chine et aux EtatsUnis. Le Sri Lanka a par ailleurs favorisé l'implantation d'une industrie nationale d'armement tournée principalement vers la production de petites unités de patrouilles navales et de véhicules blindés. Tandis que la guerre civile s'est intensifiée, ravageant l'économie du pays, les besoins en armement sont devenus plus urgents et plus variés. Parmi les acquisitions effectuées par le Sri Lanka en 1990, on trouverait notamment des transports de troupes blindés (APC) de deuxième main fournis par l'Iran et de vieux avions de transport $\mathrm{Y} 12$ chinois.

La Thaïlande qui possède en comparaison une liste d'achats beaucoup plus importante et des responsables militaires plus ambitieux et plus influents a pu quant à elle, obtenir en 1987-88 du matériel chinois en quantité suffisante pour équiper 4 divisions d'infanteries mécanisées et également des frégates (équipées de moteurs américains), des chars, des APC, des canons de campagne et anti-aériens, à un prix d'ami et à des taux d'intérêts ridiculement bas. En 1990, les Thaïlandais ont cherché à se procurer des missiles surface-surface pour leurs frégates lorsque les modèles américains se sont avérés trop coûteux. Les raisons qui ont poussé les autorités chinoises à accepter un tel marché sont d'ordre économique et stratégique, intéressées qu'elles étaient par la présence d'une Thaïlande fortement armée par rapport au Vietnam. 
La détermination des politiques gouvernementales concernant l'approvisionnement en armement est évidemment soumise à l'influence des militaires. Le discours relatif à l'accroissement de l'armée en Thaïlande en 1990, a été accompagnée par une campagne anti-corruption largement médiatisée. Chaque service armé thaïlandais disposerait, diton, de fonds secrets pour subvenir librement à ses propres dépenses. La Thaïlande, comme Sri Lanka, a favorisé aussi l'implantation d'une industrie nationale d'armement axée plus particulièrement sur la construction navale et la production d'armes légères. Cela n'a pas empêché le maintien de ses liens traditionnels avec les Etats-Unis parmi lesquels figurent un accord militaire de pré-positionnement en vertu duquel du matériel d'artillerie a été stocké au cours des années quatre-vingt pour être éventuellement utilisé contre le Vietnam. Au terme de cet arrangement la Thaïlande se voyait attribué la moitié du matériel en question et bénéficiait de nouveau crédit du FMS en 1988 et 1989. En 1990, l'armée thaïlandaise a cherché à se procurer 300 chars américains de modèle ancien, les forces aériennes ont demandé quant à elles des chasseurs F-16 et Tornado supplémentaires mais ont également envisagé de négocier pour des F-5 rénovés. Quoique les militaires thaillandais aient apprécié les prix particulièrement compétitifs de certains matériels proposés par le Brésil, comme les roquettes, une politique d'achat prudente les a conduits à faire de nouveau appel aux grands exportateurs d'armes habituels lorsque la cessation des paiements irakiens a fait peser des doutes sur la viabilité des programmes brésiliens.

La Thaillande la Malaisie et le Chili sont les plus gros consommateurs d'armes parmi les pays en voie de développement et particulièrement parmi ceux dont les doctrines militaires sont les plus avancées. A l'inverse du Nigéria dont la politique de défense territoriale est plus passive, ces pays se sont heurtés à des limitations budgétaires et à de sérieux problèmes d'endettement dans leur quête de nouveaux systèmes d'armes plus sophistiquées et plus fables. Pour s'en sortir, un bon nombre de ces états sont en passe de devenir à leur tour des producteurs d'armes au sein du tiers-monde, à l'image de la Thaillande qui grâce au patronage de diverses grandes puissances est véritablement armée jusqu'aux dents. Toutefois, ils n'ont pas abandonné pour autant leur recherche de nouvelles sources d'approvisionnement en armes et de financement, par exemple en Chine et en Israël. Citons à cet égard les frégates thaïlandaises dotées d'équipements chinois et américains, l'accord de troc entre Israël et la Colombie, ou la production par le Chili de véhicules suisses sous licence. Une grande partie du matériel est acheté de seconde main mais rénové avec les composants dernier $\mathrm{cri}^{22}$. Pour ces pays priorité est donnée à l'acquisition de sous-marins, qui passent pour être moins vulnérables et plus difficilement détectables par les systèmes de surveillance spatiale, de commandement, de contrôle et de guidage, qui ont prouvé toute leur efficacité dans la guerre des Malouines et celle du Golfe. La technologie allemande et italienne dans le domaine des sous-marins est d'ailleurs très appréciée, soit à l'achat, soit en contrat d'entreprise. La frénésie des achats d'armements semble se manifester périodiquement lorsque les militaires peuvent tirer parti des allocations budgétaires pour combler les retards accumulés lors des périodes de récessions économiques. Les pays sousdéveloppés désireux de se procurer des armes utilisent à l'heure actuelle des réseaux d'approvisionnement fort complexes et ont recours habituellement à des combinaisons ingénieuses et variées pour parvenir à leurs fins. On observe également que le Chili s'est livré à des opérations de réexportation d'obusiers israéliens et sud-africains assemblés sur place. L'abondance des sources d'approvisionnement est ainsi à l'origine 
de combinaisons multiples et ingénieuses. Fin des conflits: fin des importations d'armement?

Il est intéressant à ce propos d'observer ce qui arrive à des états qui ont été confrontés à des conflits, ou à des guerres de longue durée et dont les problèmes de sécurité semblent enfin en voie de règlement, comme c'est le cas au Yémen, au Zimbabwe, au Chili, en Algérie, et en Malaisie.

Le Zimbabwe a réduit ses forces armées d'approximativement 36000 hommes (passant de 46000 à 10.000) depuis que la menace interne et externe que faisait peser le Mozambique a diminué, mais a toujours des plans pour d'importants renforcements en matériel. En 1988, son Parlement a officialisé l'infrastructure destinée à l'approvisionnement de l'armée par la création du Comité d'équipement de défense chargé de créer des compagnies et des agences responsables de l'achat et de la vente de matériels militaires. En 1989, un accord portant sur un prêt à faible intérêt d'un montant de 102 millions de dollars a été conclu avec la Chine pour la livraison de systèmes de défense aérienne sophistiqués (comprenant des avions, des missiles, et des radars) dont le coût réel dépassait en fait largement la valeur du prêt. Ce fut la vente la plus importante d'avions de la Chine à un pays aFricain. Ajoutons enfin que même après la levée de l'état d'urgence en 1990, le budget de la défense n'a cessé d'augmenter sensiblement au profit, en particulier, de l'armée de terre.

$\mathrm{Au}$ Chili, malgré l'accord intervenu avec l'Argentine sur le Beagle Channel, les militaires sont restés assez influents pour se voir dotés d'un matériel considérable. L'armée chilienne avait de plus multiplié ses achats d'armement avant d'abandonner le pouvoir au nouveau gouvernement civil. Toujours sous le coup de l'embargo américain à la fin des années quatre-vingt, le Chili a cherché à moderniser lui-même les matériels militaires qu'il possédait (F-5 américains et Mirages français), avec l'aide d'Israël, en les équipant par exemple d'une avionique plus performante et en les armant. La marine a obtenu quant à elle des missiles de défense aérienne israéliens pour 4 destroyers de fabrication britannique. Associé à l'Espagne pour l'assemblage d'avions, le Chili est également devenu un marché très convoité pour les consortiums d'armes italobrésiliens.

Un accord conclut en novembre 1989 et par lequel il est mis fin, de manière formelle, à la rébellion du parti communiste en Malaisie (et prévoyant la destruction de ses armes) n'a pas hypothéqué un développement important de l'armée. Certaines transactions coûteuses, telles celles portant sur l'acquisition d'avions de chasse britanniques (des Tornados) ont certes été suspendues en 1990. Toutefois la plupart des dernières augmentations budgétaires sont destinées à la défense et vont concerner des dépenses d'investissement et de personnel. Face à la persistance de certains troubles régionaux, aux inquiétudes du gouvernement face à certains groupes ethniques (chinois et chrétiens), les militaires ont anticipé une stratégie de défense à long terme et misent sur leurs capacités anti-insurrectionnelles. C'est pourquoi, confrontées à l'augmentation des coûts lors des négociations sur le Tornado et les systèmes aériens de défense britanniques qui auraient conféré à l'aviation malaise un rayon d'action plus important, l'Australie mise à part, la Malaisie a envisagé l'acquisition d'autres avions britanniques (Hawk) et du matériel français. L'importante commande de la marine prévoyait entre autre l'achat d'un sous-marin, de systèmes de défense anti-aériens, de canons légers, de corvettes, de nouveaux transports de troupes et d'hélicoptères. 
Cependant la Malaisie est atypique. Pour beaucoup de pays en voie de développement dans lesquels la classe militaire a accès aux postes de responsabilité, l'acquisition de matériels à un prix raisonnable, la mobilité et la souplesse l'emportent sur le tonnage et la puissance de feu.

Le retour de l'Algérie à des relations plus détendues avec ses anciens adversaires dans la région (le Maroc et la Libye), quoiqu'elles demeurent peu cordiales, a ouvert la voie à un effort de diversification des sources d'approvisionnement en armes en vue d'échapper à une dépendance à l'égard de l'Union soviétique. En 1985, Alger qui n'a pas caché son intérêt pour l'achat ultérieur d'un avion ultramoderne, a honoré un premier contrat d'armement conclu 10 ans auparavant avec la France, a acheté un avion de transports C-130 aux Etats-Unis, puis a conclu avec le Royaume-Uni l'acquisition d'unités navales. Les demandes de l'URSS en vue de disposer de bases dans le pays et afin de procéder à des exercices militaires communs ont été rejetées. L'Algérie a reçu néanmoins de l'URSS des avions de transport en 1989. Par ailleurs un nouveau plan d'approvisionnement en armes (combinant notamment de multiples fournisseurs) semble être en voie de préparation, plan qui pourrait laisser présager le déclin des parts soviétiques dans la plupart des pays d'Afrique, même si les prix restent toujours un élément du choix d'états qui manquent de ressources pétrolières.

La place d'un état dans l'environnement régional conditionne aussi largement la question de son approvisionnement en armes comme l'illustre le cas du Yémen. La qualité des relations entretenues avec des voisins bien équipés peut soit accélérer, soit retarder cette acquisition. La chaleur des relations politiques et de l'équilibre des rivalités dans la zone constituent donc des facteurs déterminants comme le montrent les relations sino-thaïlandaises. Le recul des intérêts soviétiques au Moyen-Orient et l'accord sur l'union du Yémen a dans une certaine mesure réduit la valeur stratégique de ce pays pour les grandes puissances. Il représente cependant toujours un lieu stratégique important pour l'égypte et l'Arabie Saoudite. Les sources d'approvisionnement en matériel militaire du Nord-Yémen ont toujours été traditionnellement mieux réparties et plus équilibrées que celles du Sud-Yémen qui comptait exclusivement sur la Syrie et les pays du bloc de l'Est. Le Nord-Yémen a été le seul endroit au monde où stratèges américains et soviétiques ont partagé la même base. Le Yémen fut l'exemple type du pays équipé d'un matériel relativement sophistiqué mais disposant d'un personnel insuffisant pour l'utiliser, des pilotes taïwanais et sudcoréens durent donc volé dans la force aérienne yéménite durant les crises qui secouèrent le pays. Depuis l'union cependant, l'Arabie Saoudite s'est montrée plutôt réticente au renforcement de son assistance militaire à un pays potentiellement instable, dans la mesure également où les tribus pro-saoudienne du nord du pays paraissaient avoir été affaiblies par cet accord.

En Asie du sud-est, les signataires des divers traités, censés coopérer comme dans le cas de l'ASEAN se surveillent mutuellement lorsqu'il est question de la conception et du développement des appareils de défense. L'Indonésie et la Thaillande, voisins de la Malaisie, ont manifesté une certaine inquiétude lorsque cette dernière a renforcé son équipement militaire qui s'est considérablement accru, et ce, bien que ces trois pays aient été engagés dans des opérations communes contre les mouvements de guérillas. En avril 1990, Singapour a demandé la mise en place de mesures de libre inspection ("open inspection"), et les Philippines se sont inquiétées lorsque la Malaisie a fait montre d'ambitions navales. Même si les querelles ne prennent pas l'ampleur d'une 
crise majeure, il reste assez de problèmes épineux, comme le conflit multinational sur les îles de Spark, pour fragiliser à l'extrême le sous-système régional de cette partie du monde.

Il existe donc des modèles ou des profils d'armement applicables aux pays sousdéveloppés. Les armes lourdes (artillerie, chars d'assaut, navires de guerre) sont beaucoup plus rares dans les pays de l'Afrique subsaharienne (excepté en Afrique du Sud) que dans les pays à la topographie plus ouverte ou plus riches comme ceux du Moyen-Orient et de l'Afrique du Nord. Les missiles basés à terre semblent aussi de plus en plus populaires au Moyen-Orient. Les états latino-américains utilisent quant à eux des blindés légers, du matériel de défense anti-char, et davantage d'équipements aérien (chasseurs et hélicoptères) et naval. En Asie de l'Est les forces navales ont gagné en importance ainsi que les hélicoptères capables de pénétrer dans la jungle, les blindés éventuellement lourds mais plus souvent légers, les roquettes anti-chars, l'artillerie, l'aviation à long rayon d'action, les systèmes anti-aériens relativement sophistiqués (SAM). Les armées africaines semblent préférer les véhicules et les transports blindés, les systèmes de défense anti-char et les forces aériennes légères disposant d'un faible rayon d'action. La politique de défense du Nigéria reste orientée sur la protection du territoire national malgré la réduction de ses dépenses militaires mais manque d'un système de défense intégré.

Entre 1987 et 1990 aucun nouveau système de défense n'a été commandé malgré le soutien apporté à une production locale d'armements incluant des explosifs, des munitions, et diverses armes légères. Le Sénégal et le Zimbabwe comptent sur leurs véhicules blindés, leurs transports de troupes blindés (APC) d'attaque au sol. Le Sénégal enfin a maintenu une petite formation de patrouille côtière et une flotte amphibie.

Plusieurs modèles peuvent se dégager des projets d'acquisition et de distribution d'armes parmi les pays en voie de développement. D'une manière générale, il apparaît que l'atténuation des tensions internationales ne se traduit pas nécessairement par une moindre propension à s'armer. Les plans d'approvisionnement sont généralement établis pour des périodes relativement longues ( 8 ans pour le Chili). Même si des projets viennent à être annulés ou suspendus, comme ce fut le cas notamment pour les achats de matériels britanniques par la Malaisie, c'est pour faire place le plus souvent à des accords d'achats de matériels de second choix, d'occasion ou rénovés. On constate que les pays sous-développés seront de plus en plus enclins à combiner ou tenter de combiner des éléments obtenus auprès de divers fournisseurs, en des systèmes d'armes uniques et améliorés. Le travail sera effectué la plupart du temps dans les ateliers du pays, en liaison avec d'autres entreprises et avec une assistance technique étrangère. Les variantes ainsi produites pourront être destinées à la réexportation dans certains cas, comme celui Chili.

Si les performances de ces technologies "exotiques" restent généralement limitées, une production de matériel proche des modèles haut de gamme sera possible. Ainsi en va-til des systèmes de vecteurs, y compris les missiles de croisière, les matériels antichars, des sous-marins généralement de taille modeste mais parfois relativement importante (Chili, Malaisie, Thaïlande), des défenses antiaériennes. Néanmoins une certaine disparité (la taille et la mobilité des armes d'une même région) subsistera probablement. Certains regroupements régionaux, comme en Péninsule Arabique, en Afrique du Nord, dans l'Asie du Sud-Est, peuvent entraîner un partage des rôles en matière d'approvisionnement et de standardisation des armements, en partie pour 
permettre à certains acteurs d'exercer un certain contrôle sur une éventuelle course aux armements et sur des voisins potentiellement instables.

Le financement restera toujours un problème, mais là encore, s'il existe une volonté réelle de se procurer des armes, il existera probablement un moyen pour les obtenir, soit par accords de troc, soit par achats à prix réduit avec obtention de prêt à taux d'intérêts réduits auprès des états exportateurs relativement pauvres, soit en faveur d'états amis représentant un intérêt stratégique, ou par des accords de co-productions, soit enfin à titre de compensation. En général le commerce international est moins porté sur la réalisation de profits engendrés sur des transactions spécifiques que sur la dynamisation de la production, de nature à favoriser les économies globales. C'est pourquoi beaucoup de fournisseurs d'armes sont tentés de vendre même à perte si les handicaps technologiques sont surmontables.

Certains pays sous-développés comme le Nigeria et, dans une certaine mesure, la Malaisie et le Sénégal ont fait preuve d'une admirable retenue dans leurs achats d'armements pendant les périodes de récession économique. Par ailleurs la défense collective ou les schémas de sécurité, comme ceux auxquels a participé le Nigeria en Afrique se sont avérés efficaces en limitant durablement les carnages. En plaçant leur politique d'armement dans une perspective d'intervention multilatérale plutôt qu'unilatérale, ces pays favorisent la paix et économisent de l'argent. Dans cette optique, Robert McNamara a proposé en 1991 de refuser l'assistance internationale au développement des pays dont les dépenses militaires excèdent un certain niveau optimal ${ }^{23}$.

Pour Thee ${ }^{24}$, les laboratoires de développement technologique des grandes puissances sont directement responsables des tendances au surarmement. Cependant la technologie représente également pour les pays nouvellement industrialisés et les pays les moins développés un attrait symbolique, attrait stimulé à la fois par la perception de la menace, comme celle que fait peser l'activité de sous-marins relativement indétectables, et par la recherche d'un certain statut, comme les militaires du Chili et de Thaïlande en donnent un exemple. L'armement a fini par être un repère technologique (quick fix) par rapport auquel se situent les échanges entre les états plus ou moins développés ainsi qu'on le voit dans les projets d'amélioration, de transformation et de rénovation de chars et d'avions. L'importance des bénéfices escomptés est sans doute exagérée mais il n'empêche que l'armement figure parmi les quelques rares activités dans le cadre desquelles coopèrent les ingénieurs des pays industrialisés et des états en voie de développement. Tant que les transferts de technologie ne s'effectueront pas de façon spontanée, tant qu'un nombre important d'ingénieurs compétents éventuellement "dissidents" devra être employé, aussi longtemps que les menaces pour la sécurité nationale ou régionale pourront être identifiées - ou inventées de toutes pièces - les armes seront perçues par les gouvernements des pays les moins développés comme un objet commercial des plus désirables. Ils chercheront à s'approprier des éléments de technologie avancée même si cela passe par l'acquisition de matériels âgés ou de second ordre. Des efforts doivent être engagés pour impliquer autant que possible les pays sources d'approvisionnement, pour éviter la surdépendance et pour détecter les réseaux d'approvisionnement, afin de dégager un maximum d'idées et de nouvelles techniques de contrôle. I1 existera, de toute façon, suffisamment d'équipements de fabrication locale, essentiellement des modèles copiés, pour que l'identification des producteur-distributeurs deviennent à la fois plus difficile et moins importante. Et si les niveaux d'endettement continueront à 
entraver la progression du marché des armes, seule une combinaison de garanties de sécurité régionale, contresignées par les grandes puissances, et un accès certain à des alternatives crédibles de technologies pourront mettre un terme définitif aux flux d'armement.

\section{NOTES}

1. Richard Grimmet, Trends in Conventional Arms Tranfers to the Third World by Major Supplier, 1982-1989, Washington, Dc , Congressional Research Service, 19juin 1990. Voir aussi Stéphanie Neuman, "Continuities and Change in the International Arms trade", Presentation of the Annual Meeting of the International Studies Association, Washington, Dc, avril 1990. CATT=Conventional Arms Transfers Talks. 2. Patrick E. Tyler, As the Dust Settles, Attention Turns to New Arms Sales", The New York Times, 24 mars 1991.

3. Les avertissements répétés de la banque EXIM à l'intention de la mauvaise utilisation par l'Irak des crédits pour des constructions militaires ont été ignorés par le Département détat américain. Cf Kaslow, 1991.

4. Lora Lumpe, Arms Sales Monitor, Washington : Federation of American Scientists, avril 1991, p. 1, citant Anthony Beilenson ; David Silverberg, "U.S. Sees Need for Mideast Arms Control in Gulf War's Wake ", Defense News, 6 mai 1991, pp. 16 et 36 ; David D. Newsome, "Is a Window Open for Arms Control ?", The Christian Science Monitor, 8 mai 1991, p. 18 ; Spurgeon Jr. Keeny, "Arms Sales : The New World Order ?" Arms Control Today (21), avril 1991, p. 2 ; Richard J. Durbin, "Taxpayers Shouldn't Underwrite US Arms Sales", The Christian Science Monitor, avril 1991.

5. Lora Lumpe, op. cit., citant James Lemunyon

6. David Silverberg, op. cit., p. 36.

7. Lora Lumpe, op. cit., citant Galia Golan.

8. Michael Wines, "Third World Seeks Advanced Arms", The New York Times, 26 mars 1991, p. A6.

9. Theresa Hitchens, "Support Grows for Joint Policy on EC Defense", 6 mai 1991, p. 37. 10. Frederic S. Pearson, "European Security Policy and Franco-German Linkage", Presented to Conference on New Thinking about European Security : Rethinking Defense Strategies, Columbia, MO, mars 1991.

11. Michael Wines, op. cit., voir aussi Marek Thee, "The Post-GulfWar Technological Armaments Spiral. Paper prepared for the East-West Workshop on Conversion Research, Berlin, avril 1991.

12. Lora Lumpe, op. cit., citant Andrew Pierre.

13. Les pays nouvellement industrialisés, avec le développement de leurs industries de défense peuvent aussi être intéressés, ou le contraire, par le contrôle du commerce des armes conventionnelles ou non conventionnelles, cela dépend en effet de leurs ambitions d'exportateurs et de leurs besoins en technologie ou en pièces détachées importées (voir Lumpe, citant Golan, 1991). 
14. John F Fialka, "Czechoslovakia Plans to Export 5500 weapons", The New York Times, 9 mai 1991, p. A8.

15. Carol Evans, "Reappraising Third-World Arms Prodruction", Survival, 28 février 1986, pp. 99-118 ; Rajesh Rajagopalan, "The developing Countries as Arms Suppliers", Strategic Analysis (12), février 1989, pp. 1323-1334.

16. Mohammed Ayoob, "The Third World in The System of States : Acute Schizophrenia or Growing Pains ?", International Studies Quarterly (33), pp. 70-71.

17. Howard La Franchi, "Algerians Defend Nuclear Program", The Christian Science Monitor, 3 mai 1991, p. 6.

18. Edward A Kololziej et Frederic S. Pearson, "The Political Economy of Making and Marketing Arms : A Test for the Systemic Imperatives of Order and Welfare", Occasional Papers (8904), St Louis, Center for International STudies, University of Missouri

19. Frederic S. Pearson, "The Correlates of Arms Importation", Journal of Peace Research (26), mai 1989, pp. 153-163 ; Peter N. Hess, "Force Ratios, Arms Imports and Foreign Aid Receipts in the Developing Nations", Journal of Peace Research, (26), novembre 1989, pp. 399-412.

20. Frederic S. Pearson, 1989, op. cit.

21. Gregory R. Copley, Defense and Foreign Affairs Handbook, 1990-91, Alexandria, VA International Media.

22. "Retrofitted", dans le texte anglais : modernisation d'un matériel par l'introduction de nouveaux systèmes d'armes sur une cellule aérienne ou changement des composants électroniques (NDT).

23. David R. Francis, "Foreign Aid Seen us Lever to Curb Armaments in Developing Nations", The Christian Science Monitor, 2 mai 1991, pp. 1-2.

24. Marek Thee, op. cit.

\section{RÉSUMÉS}

Les projets pour limiter le commerce des armes se sont multipliés avee l'achèvement d'un bon nombre de contlits régionaux et la fin de la guerre froide. La guerre du Golfe a également fait évoluer les conditions de sécurité des pays les moins développés, et les principaux vendeurs d'armes ont diminué leurs aides économiques à l'exportation des armes. A partir de l'examen de nombreux cas régionaux, l'auteur conclut que l'avenir des politiques d'armement des pays sousdéveloppés dépend de l'ordre international, des effets de la guerre du Golfe, des conditions économiques et de leurs perceptions de la menace interne ou externe.

Prospects for limitations of global arms sales improved with the settlement of a number of regional disputes and the end of the Cold War. The 1991 Gulf War also stirred new security concerns among less developed countries (LDCs), and the major powers limited their economic incentives to export weapons. Based on a review of several regional cases, the article infers that the prospect for continued LDC armament appears to depend on international hierarchies, recent war experiences, domestic and foreign threats (decline in the latter not necessarily diminishing 
weapon demands), and economic contexts. An evident pattern is the refitting and upgrading of existing equipment in the search for higher technology.

INDEX

Index géographique : Pays du Sud (Tiers Monde), Relations Nord/ Sud

Mots-clés : armes et armement, pauvreté, violence, contrebande et trafic

\section{AUTEUR}

\section{FREDERIC S. PEARSON}

Directeur du Centre d'études sur la paix et les conflits, Professeur de science politique de Wayne State, Detroit, USA 